\title{
Les déchets radioactifs Leur gestion industrielle
}

\author{
J.M. LAVIE $\left({ }^{\star}\right)$ \\ (Manuscrit reçu le 7 juillet 1982)
}

\begin{abstract}
RÉSUMÉ
Cette présentation est une introduction à une série d'articles destinée à faire le point sur la situation actuelle en matière de gestion a long terme des déchets radioactifs. L'auteur y définit ce que l'on entend par gestion industrielle des déchets radioactifs et en formule les objectifs. Cette brève esquisse est complétée par un aperçu des données du problème français.
\end{abstract}

\section{ABSTRACT}

This paper introduces a series that will review the present situation in the field of long-term management of radioactive wastes. Both the meaning and the purposes of an industrial management of radioactive wastes are specified. This short introduction is complemented by an outline of data on the French problem.

La production d'énergie électronucléaire et l'utilisation de radioisotopes, comme toute activité humaine, engendrent la production de déchets. Il convient donc, à un moment donné, de les gérer industriellement.

Traditionnellement, et conformément d'ailleurs à la loi de 1975, on appelle déchet d'une opération industrielle les sous-produits qui ne peuvent pas ou ne peuvent plus être utilisés. Ces déchets sont considérés comme radioactifs lorsque leur activité est supérieure à un seuil fixé par les autorités de sûreté.

Il est à noter que, si l'industrie nucléaire produit des déchets radioactifs, l'utilisation des radioéléments dans l'industrie, les hôpitaux, les laboratoires, très répandue et actuellement en expansion, laisse également, après utilisation, des déchets dont certains sont radioactifs. Par contre, les combusbustibles irradiés sortant des centrales nucléaires ne sont pas des déchets, contrairement au vocabulaire souvent employé, puisqu'ils contiennent des produits rendus utilisables par l'opération du retraitement.

(*) Agence nationale pour la gestion des déchets radioactifs (ANDRA) CEA, 31-33, rue de la Fédération, 75015 Paris. 
La gestion industrielle des déchets radioactifs est l'ensemble des opérations et des mesures qui, depuis leur production jusqu'à leur élimination définitive, en passant par leur traitement et leur conditionnement, assurent avec la technologie accessible et dans des conditions économiques raisonnables, une protection satisfaisante des travailleurs et des générations présentes ou futures contre les risques éventuels présentés par ces déchets. La gestion des déchets radioactifs commence dès leur production, et même bien auparavant si l'on y intégre les choix technologiques des processus qui aboutissent à leur création.

II est de pratique courante de distinguer:

- la gestion en amont de l'entreposage et du stockage, c'est-à-dire la production, la récupération, le tri, le traitement et le conditionnement des déchets sous forme solide en des colis élaborés;

- la gestion en aval du conditionnement, qui aboutit à l'évacuation de ces colis vers les installations appropriées de stockage à moyen ou long terme.

C'est essentiellement pour faire face au deuxième volet qu'à été créée I'ANDRA, Agence nationale pour la gestion des déchets radioactifs, qui ne saurait cependant se désintéresser du premier, compte tenu des étroites interrelations entre les deux aspects de la gestion.

Chargée, en application de la politique définie par le gouvernement, des opérations de gestion à long terme des déchets radioactifs, l'ANDRA a quantifié l'évolution des besoins dans ce domaine et dégagé un plan d'action permettant d'apporter, en temps voulu, les solutions aux problèmes posés. Les solutions techniques proposées s'appuient d'une part sur les connaissances acquises, d'autre part sur un important programme de confirmation et de développement de celles-ci en vue notamment d'optimiser l'ensemble du système. Certaines de ces solutions techniques sont déjà mises en pratique, les autres peuvent et doivent dépasser maintenant le stade de la conception pour atteindre celui de la réalisation, au moins à titre de démonstration.

Les problèmes posés par la gestion industrielle à long terme des déchets radioactifs ne diffèrent pas, dans leur essence, de ceux de la gestion de tous les autres déchets, avec cependant des avantages notables: leur très faible volume, leur décroissance radioactive et la référence à la radioactivité naturelle que peuvent envier nombre de déchets chimiques toxiques entièrement nouveaux sur terre.

Les objectifs de cette gestion qui doit, outre la protection de l'homme d'aujourd'hui et de demain, préserver son environnement et limiter la charge des générations futures, seront atteints:

- au plan de la prévention : par une limitation au strict minimum de la production des déchets et de leur activité par leur tri, et une éventuelle réduction de volume;

- au plan de la protection: par l'interposition de systèmes de barrières entre ces déchets et l'environnement afin d'assurer leur confinement pendant la durée nécessaire à la décroissance de leur radioactivité ou de limiter leur diffusion vers l'environnement. 
La mise en œuvre de ces principes au plan industriel demande :

- de recenser et d'identifier les besoins, ce qui est l'objet des prévisions de production et de livraison des déchets, prévisions qui doivent porter non seulement sur les volumes, mais aussi sur la nature des déchets, ainsi que sur les délais d'évacuation;

- de définir des concepts de gestion à la fois accessibles sur le plan technologique, raisonnables sur le plan économique, satisfaisants sur plan de la sûreté et de la protection et suffisamment simples et clairs pour être aisément compris et acceptés par l'opinion publique. Cette définition des concepts doit tenter d'optimiser et de réduire tant la radioexposition des travailleurs, que celle des populations présentes ou à venir ;

- de choisir des sites, ou tout au moins des types de sites possibles, répondant aux critères de choix résultant des concepts d'évacuation retenus pour y implanter le ou les centres de stockage à long terme. C'est ce choix et non seulement le volume des déchets radioactifs qui constitue, en fait, le goulet d'étranglement à leur évacuation.

Cette première approche prospective réalisée, et bien acceptée, à la fois par les autorités de tutelle et l'opinion publique, il devient alors possible de définir de manière cohérente et optimisée :

- les spécifications techniques auxquelles doivent répondre le conditionnement et l'emballage des différentes catégories de déchets, en vue de leur prise en charge par l'ANDRA pour évacuation vers les centres de stockage à long terme. Chaque type de colis fait l'objet d'une procédure d'agrément par I'ANDRA. Le dossier d'agrément comporte, outre les caractéristiques du colis et l'origine des déchets contenus, le livre de procédé concernant sa réalisation ;

- l'organisation de l'assurance qualité et les contrôles de qualité que l'ANDRA fait ou fera exécuter pour s'assurer de la conformité d'une part des colis au dossier d'agrément et d'autre part des centres de stockage aux spécitications de construction et d'exploitation.

Par ailleurs, de cette approche prospective, se dégage ou se dégagera un plan de recherche et de développement appliqué à la gestion à long terme des déchets dont il convient ou conviendra de s'assurer que les objectifs et les délais sont bien couverts par les programmes nationaux ou multinationaux.

Pour atteindre les objectifs définis précédemment, il est nécessaire d'intervenir et de mettre en œuvre des processus adaptés tout au long du cycle de gestion des déchets, en particulier au niveau de la production des déchets, de leur identification et de leur tri, de leur traitement et conditionnement, de leur entreposage éventuel, de leur stockage à long terme.

L'ensemble des travaux effectués au cours des 10 à 20 années précédentes a permis de mettre au point dans chacun de ces domaines des techniques fournissant des solutions variées permettant d'optimiser progressivement l'ensemble du cycle de gestion.

Ces mises au point ont été facilitées par une coopération internationale très active, qui a permis également de dégager un certain consensus. 
Outre les échanges bilatéraux plus ou moins codifiés, tels que les accords du CEA avec la coopérative suisse pour l'entreposage des déchets radioactifs (CEDRA), d'imortants travaux sont effectués sous l'égide d'organisations internationales : les Communautés économiques européennes pour la faisabilité du stockage profond, l'OCDE pour le stockage dans les fonds sous-marins (programme sea-bec) et les conditions de stockage dans les formations cristallines (mine de fer suédoise désaffectée de Stripa).

\section{Les données du problème français}

L'expérience acquise en France et à l'étranger permet actuellement de prévoir avec une précision suffisante la production de déchets des différentes installations nucléaires existantes ou en projet. suivant:

La synthèse de ces prévisions, pour la France, est fournie par le tableau

\begin{tabular}{|c|c|c|c|}
\hline & & $\begin{array}{c}\text { Quantité cumulée } \\
\text { en } 1982\end{array}$ & $\begin{array}{c}\text { Quantité cumulée } \\
\text { en } 2000\end{array}$ \\
\hline A & $\begin{array}{l}\text { Déchets de faible } \\
\text { et moyenne activité }\end{array}$ & $170000 \mathrm{~m}^{3}$ & $800000 \mathrm{~m}^{3}$ \\
\hline B & Déchets alpha & $10000 \mathrm{~m}^{3}$ & $45000 \mathrm{~m}^{3}$ \\
\hline & Déchets vitrifiés & $120 \mathrm{~m}^{3}$ & $3000 \mathrm{~m}^{3}$ \\
\hline
\end{tabular}

L'ANDRA, responsable de la gestion à long terme des déchets radioactifs, dispose d'un centre de stockage de surface des déchets de faible et moyenne activité à période courte, qui sont les seuls déchets radioactifs dont le stockage définitif soit autorisé en France. Ce centre, appelé Centre de la Manche, contigu à l'installation de retraitement de la Hague, offre avec une superficie de 12 ha une capacité d'accueil de l'ordre de $400000 \mathrm{~m}^{3}$ dont $150000 \mathrm{~m}^{3}$ environ sont déjà utilisés. La cadence annuelle de livraison de cette catégorie de déchets au Centre de la Manche est actuellement de $20000 \mathrm{~m}^{3}$.

Les autres catégories de déchets sont entreposées sur les sites de production en attendant les décisions gouvernementales sur le programme général français de gestion des déchets radioactifs qui devrait permettre la mise en place de structures opérationnelles au début de la prochaine décennie.

Quel est le coût de l'évacuation des déchets?

Peut-il mettre en péril la rentabilité de l'électronucléaire?

Les conclusions convergentes des études économiques effectuées sont rassurantes. Ce coût ne dépasse pas quelques pour cent du prix de revient du kwh nucléaire. Ce faible pourcentage ne doit pas cependant masquer qu'en valeur absolue, en raison de l'importance de la contribution attendue de l'élecnucléaire dans la production future d'énergie il conduit, appliqué au coût de la production cumulée d'énergie jusqu'en l'an 2000, à des sommes de l'ordre de plusieurs dizaines de milliards de francs. 
Le financement de cette gestion est assuré par les producteurs de déchets.

\section{Conclusion}

Cette brève esquisse, qui sera complétée par des articles reprenant plus en détail certains points, notamment les objectifs de protection, les options de sûreté, les concepts de stockage, l'expérience du Centre de la Manche et les garanties de bonne fin, permet déjà de délimiter le cadre dans lequel s'inscrit la gestion industrielle des déchets radioactifs.

La mise en place de moyens industriels adéquats, reposant sur des techniques étudiées et développées dès le début de l'ère nucléaire, et le choix de sites adaptés permettront de résoudre le problème de l'évacuation des déchets radioactifs dans des conditions de sécurité rivalisant largement avec celles de l'évacuation des autres déchets industriels. Ils permettront, également, de renforcer auprès du public la crédibilité des solutions retenues, d'autant plus que l'effort de recherche entrepris dans le domaine du traitement et du stockage sera une garantie supplémentaire. 